\title{
Effects of long-term inhaled corticosteroids on skin collagen synthesis and thickness in asthmatic patients
}

\author{
K. Haapasaari*, O. Rossi**, J. Risteli+, A. Oikarinen*
}

Effects of long-term inhaled corticosteroids on skin collagen synthesis and thickness in asthmatic patients. K. Haapasaari, O. Rossi, J. Risteli, A. Oikarinen. OERS Journals Ltd 1998.

ABSTRACT: There are only a few studies on the adverse effects of inhaled corticosteroids on the skin in asthmatic patients. Therefore, we evaluated the effect of inhaled corticosteroids on de novo collagen synthesis of skin and bone, skin thickness and the total amount of skin collagen.

Twenty seven consecutive new asthmatic patients, on a moderate dose of budesonide or beclomethasone dipropionate, were invited to take part in this prospective study. Radioimmunological analyses of aminoterminal propeptides of type I and III procollagens (PINP, PIIINP, respectively) in suction blister fluid (SBF) of skin and in serum and carboxyterminal propeptide of type I procollagen (PICP) and cross-linked carboxyterminal telopeptide of type I collagen (ICTP) in serum were performed at entry and after 3 and 6 months of inhaled corticosteroid treatment. Ultrasound measurements of skin thickness at two sites were performed at entry, at 3 and 6 months and after 1-2 yrs of inhaled corticosteroid treatment in 20 patients, six of whom had been prescribed one or more courses of oral corticosteroids. Skin hydroxyproline of punch biopsies was determined to measure the total amount of skin collagen (males, at entry and at 6 months).

Skin thickness and the total amount of skin collagen on the abdomen were unchanged after 1-2 yrs of inhaled corticosteroid use. A slight decrease was observed in the upper arm skin thickness, especially in those subjects who had received inhaled plus oral corticosteroids. The procollagen propeptide concentrations (PINP, PIIINP) were markedly decreased in SBF at 3 months and remained at this level at 6 months. In serum, a slight decrease was seen in the PINP, PIIINP and ICTP concentrations at 3 and 6 months.

In conclusion, inhaled corticosteroids decrease the collagen synthesis of skin and bone, but skin thickness and the total amount of collagen in skin are not changed markedly after 1-2 yrs of treatment.

Eur Respir J 1998; 11: 139-143.
Depts of *Dermatology, **Internal Medicine and ${ }^{+}$Clinical Chemistry, University of Oulu, Oulu, Finland.

Correspondence: A. Oikarinen

Dept of Dermatology

University of Oulu

Kajaanintie 50

FIN-90220 Oulu

Finland

Fax: 0035883153135

\section{Keywords: Bone}

inhaled corticosteroids procollagen propeptides skin

Received: February 121997

Accepted after revision October 41997

This study was supported by the Medical Research Council of the Academy of Finland.
Inhaled corticosteroids (ICs) have been recommended as first-line therapy for all but the mildest forms of asthma. The treatment is likely to be long and the question of safety is important. The adverse effects associated with this treatment are well known, such as thrush, dysphonia, sore throat, changes in lipid and bone metabolism, growth retardation and cataract [1]. Furthermore, ICs also have an effect on skin. Six week therapy with ICs is sufficient to repress collagen synthesis of skin [2]. Purpura and dermal thinning have been associated with high-dose IC treatment [3].

Collagen is the most abundant protein in humans. Type I collagen comprises $90 \%$ of the bone organic matrix, and in skin it is the major structural component of dermis, most of which consists of type I collagen (80\%), the rest being mostly type III collagen (10-15\%) [4].

Collagen is synthesized in a precursor form, procollagen. During synthesis, procollagen propeptides are cleaved off by specific proteases enabling collagen molecules to assemble into functional fibres [5]. Thus, the amount of these propeptides reflects de novo synthesis of collagen.

Radioimmunological analysis of procollagen propeptides in serum [6-8] and extracellular fluid [9] is a new clinical tool for monitoring the collagen synthesis rate in various clinical conditions, including the effect of topical and systemic corticosteroid treatments [9, 10]. We have shown recently that the decrease of collagen propeptides in skin suction blister fluid (SBF) is associated with a decline of type I collagen messenger ribonucleic amino acid (RNA) levels (unpublished data).

There have been no studies assessing simultaneously both the synthesis and the net amount of collagen in skin in asthma patients receiving ICs. We therefore studied the effect of IC treatment on collagen synthesis and the amount of collagen in skin and the collagen synthesis and degradation in bone in asthma patients. A special emphasis was placed on the evaluation of the risk of developing clinical skin atrophy in patients treated with ICs. 


\section{Materials and methods}

\section{Subjects}

Twenty seven newly diagnosed asthmatics (using the criteria of the American Thoracic Society [11]) were invited to take part in this prospective study. For the treatment of asthma, a moderate dose (800-1000 $\mu \mathrm{g}$ ) of inhaled corticosteroid (budesonide, BUD, or beclomethasone dipropionate, BDP) was prescribed. Two patients received a higher dose of BUD (1200 $\mu \mathrm{g}$ and $1600 \mu \mathrm{g}$, respectively). Twenty three patients were treated with BUD. Nineteen of them used Pulmicort ${ }^{\circledR}$ Turbuhaler ${ }^{\circledR}$ (Astra, Espoo, Finland) and four used Cortivent ${ }^{\circledR}$ (Leiras, Turku, Finland) inhalation aerosol with a spacer, Rondo® (Leiras). Three patients treated with BDP (Becotide $\AA$, Glaxo Wellcome, Espoo, Finland) had a spacer, Volumatic ${ }^{\circledR}$ (Glaxo Wellcome), and one patient was treated with inhalation powder (Becotide Rotadisk®, Glaxo Wellcome). All patients also used an inhaled $\beta_{2}$-agonist as needed. None had previously been on inhaled or topical corticosteroids, or had diseases affecting collagen metabolism. Table 1 shows the initial data.

A written informed consent was obtained from all the patients. This study was approved by the Ethics Committee of the Faculty of Medicine and the Oulu University Hospital.

\section{Study design}

Skin thickness was measured with ultrasound (Dermascan A, Cortex technology, Hadsund, Denmark) on the abdomen and the upper right arm before the treatment and at 3 and at 6 months. Three individual measurements were made, and the mean was calculated at each site. At these time-points, suction blisters were induced on the abdominal skin using a disposable suction blister device (Dermouac ${ }^{\circledR}$, Ventipress, Lappeenranta, Finland) for the majority of patients during the follow-up and a plastic suction blister device for some patients at entry [12]. Blister fluid was collected and kept frozen until the radioimmunological assays of aminoterminal propeptides of type I and III procollagens (PINP, PIIINP, respectively) [6, 8]. Serum samples were obtained at the baseline and at 3 and 6 months. From these samples, carboxy- and aminoterminal propeptides of type I procollagen (PICP, PINP, respectively) [6,7], PIIINP [8] and cross-linked carboxyterminal telopeptide of type I procollagen (ICTP) [13] were analysed using radioimmunological assays.

Table 1. - Patients and the initial treatment

\begin{tabular}{lc}
\hline & Patients \\
\hline Sex male/female & $\mathrm{n}$ \\
Age $^{+}$yrs & $19 / 8$ \\
Smoking & $38(16-68)$ \\
$\quad$ Nonsmoker & 7 \\
$\quad$ Former & 16 \\
$\quad$ Current & 4 \\
Inhaled corticosteroids & \\
$\quad$ BUD 800 $\mu \mathrm{g}$ & 22 \\
BUD $1600 \mu \mathrm{g}$ & 1 \\
BDP 800 $\mu \mathrm{g}$ & 1 \\
$\quad$ BDP $1000 \mu \mathrm{g}$ & 3 \\
\hline
\end{tabular}

+: mean (range).
Skin punch biopsies (diameter $4 \mathrm{~mm}$ ) were taken from the abdominal skin of 20 male subjects at the baseline and at 6 months. From these biopsies, skin hydroxyproline was determined using a colorimetric method [14].

After 1-2 yrs of IC use, the subjects were invited to attend for a further measurement of skin thickness. Twenty of them consented, and skin thickness was measured on the upper arm and the abdominal skin by ultrasound. Six of these patients had received one or more courses of oral corticosteroids after the initial 6 month follow-up.

As controls, skin thickness of upper arm, serum PINP, PIIINP and ICTP levels and PINP and PIIINP values of blister fluid of 14 healthy females (median age $51 \mathrm{yrs}$, range 47-54 yrs) were followed for 6 months using the same method. PINP and PIIINP in SBF were also followed in eight male volunteers for $1 \mathrm{yr}$ (median age $24 \mathrm{yrs}$, range $22-32$ yrs).

\section{Statistical analysis}

A logarithmic transformation of the original values (procollagen propeptide concentrations) was taken to obtain a more Gaussian-like distribution. For the statistical analysis, paired-sample t-test Statistical Products and Service Solutions (SPSS) for Windows was used to compare the values obtained at 3 and 6 months to those obtained at entry.

\section{Results}

\section{Skin thickness and skin hydroxyproline}

Considering all 27 subjects, abdominal skin thickness did not change during the 6 month follow-up (table 2). On the upper arm, a slight decrease was seen (mean $0.09 \mathrm{~mm}$, $95 \%$ confidence interval $(\mathrm{CI}) 0.04-0.14 \mathrm{~mm}, \mathrm{p}=0.001)$ at 6 months (females plus males). In females, the decrease was $0.05 \mathrm{~mm}(95 \%$ CI $0.003-0.105 \mathrm{~mm}, \mathrm{p}=0.041)$ and in males $0.1 \mathrm{~mm}(95 \%$ CI $0.031-0.167 \mathrm{~mm}, \mathrm{p}=0.007)$.

One to two years after the beginning of the study, the skin thickness of 20 patients was measured on the abdomen and the upper arm. Fourteen patients had used IC treatment only. The skin thickness of the abdomen and the

Table 2. - The skin thickness on the upper arm and abdomen, aminoterminal propeptides of type I (PINP) and III (PIIINP) procollagens in suction blister fluid of skin and skin hydroxyproline

\begin{tabular}{|c|c|c|c|}
\hline & \multicolumn{3}{|c|}{ Time after treatment months } \\
\hline & 0 & 3 & 6 \\
\hline Skin thickness mm & & & \\
\hline Upper arm & $\begin{array}{c}1.64 \\
(1.14-1.95)\end{array}$ & $\begin{array}{c}1.56 \\
(1.15-1.95)\end{array}$ & $\begin{array}{c}1.50 \\
(1.11-1.87)\end{array}$ \\
\hline Abdomen & $\begin{array}{c}2.01 \\
(1.16-2.54)\end{array}$ & $\begin{array}{c}2.00 \\
(1.16-2.52)\end{array}$ & $\begin{array}{c}2.00 \\
(1.12-2.57)\end{array}$ \\
\hline PINP $\mu \mathrm{g} \cdot \mathrm{L}^{-1}$ & $\begin{array}{c}557 \\
(70-4580)\end{array}$ & $\begin{array}{c}134 \\
(40-1158)\end{array}$ & $\begin{array}{c}167 \\
(28-1045)\end{array}$ \\
\hline PIIINP $\mu \mathrm{g} \cdot \mathrm{L}^{-1}$ & $\begin{array}{c}244 \\
(16-1580)\end{array}$ & $\begin{array}{c}38 \\
(12-1040)\end{array}$ & $\begin{array}{c}60 \\
(7-620)\end{array}$ \\
\hline $\begin{array}{l}\text { Skin hydroxyproline } \\
\mu \mathrm{g} \cdot \mathrm{mm}^{-2}\end{array}$ & $\begin{array}{c}10 \\
(6-23)\end{array}$ & ND & $\begin{array}{c}12 \\
(7-15)\end{array}$ \\
\hline
\end{tabular}

Values are expressed as median (range in parenthesis). The data from female and male patients are combined. ND: not determined. 
upper arm was unchanged compared to the measurement at entry or at 6 months. The mean (range) abdominal skin thickness of these 14 patients was $2.06 \mathrm{~mm}$ (1.75-2.54 $\mathrm{mm})$ at entry, $2.06 \mathrm{~mm}(1.75-2.55 \mathrm{~mm})$ at 6 months and $2.09 \mathrm{~mm}(1.74-2.91 \mathrm{~mm})$ after $1-2$ yrs. The mean (range) upper arm skin thickness was $1.56 \mathrm{~mm}$ (1.22-1.95 mm) at entry, $1.50 \mathrm{~mm}(1.14-1.86 \mathrm{~mm})$ at 6 months and 1.55 $\mathrm{mm}(1.06-2.08 \mathrm{~mm})$ after $1-2$ yrs.

Six patients, who had received IC plus oral corticosteroid treatment for one to several weeks after the 6 month follow-up, showed thinning of the skin on the upper arm (table 3). No such change was observed in abdominal skin thickness.

Table 3 shows the individual upper arm skin thickness values and PINP concentrations before the beginning of IC treatment and during the follow-up in male and female patients separately. The initial values of upper arm skin thickness and PINP in SBF were lower in females than in males. The ratio of upper arm skin thickness in females versus males was 0.82 . In control females, median (range) skin thickness of the upper arm was $1.43 \mathrm{~mm}(1.05-2.00$ $\mathrm{mm})$ at entry and $1.37 \mathrm{~mm}(1.03-2.05 \mathrm{~mm})$ at 6 months. These values are close to those found in IC-treated females (see table 3).
The total amount of skin collagen, expressed as skin hydroxyproline $\mu \mathrm{g} \cdot \mathrm{mm}^{-2}$, did not change during the 6 month follow-up (table 2).

\section{Collagen propeptides in SBF in males and females}

PINP had decreased by $66 \%(95 \%$ CI $51-77 \%, \mathrm{p}<$ $0.001)$ at 3 months and by $70 \%$ (95\% CI 54-80\%, p< $0.001)$ at 6 months. PIIINP had decreased by $76 \%(95 \%$ CI $60-86 \%, \mathrm{p}<0.001)$ at 3 months and by $76 \%(95 \% \mathrm{CI}$ $62-85 \%, \mathrm{p}<0.001)$ at 6 months. The medians and ranges of the propeptide concentrations in SBF are shown summarized in table 2. The individual values for PINP for males and females separately are shown in table 3. In female controls, the median (range) of PINP levels in SBF was $175 \mu \mathrm{g} \cdot \mathrm{L}^{-1}\left(48-362 \mu \mathrm{g} \cdot \mathrm{L}^{-1}\right)$ at entry and $273 \mu \mathrm{g} \cdot \mathrm{L}^{-1}$ $\left(59-593 \mu \mathrm{g} \cdot \mathrm{L}^{-1}\right)$ after 6 months. The corresponding PIIINP levels were $58 \mu \mathrm{g} \cdot \mathrm{L}^{-1}\left(20-208 \mu \mathrm{g} \cdot \mathrm{L}^{-1}\right)$ at entry and 127 $\mu \mathrm{g} \cdot \mathrm{L}^{-1}\left(14-213 \mu \mathrm{g} \cdot \mathrm{L}^{-1}\right)$ after 6 months.

In male controls, the median (range) of PINP in SBF was $216 \mu \mathrm{g} \cdot \mathrm{L}^{-1}\left(136-705 \mu \mathrm{g} \cdot \mathrm{L}^{-1}\right)$ at entry and $236 \mu \mathrm{g} \cdot \mathrm{L}^{-1}$ (87-844 $\mu \mathrm{g} \cdot \mathrm{L}^{-1}$ ) after $1 \mathrm{yr}$. The median (range) of PIIINP was $58 \mu \mathrm{g} \cdot \mathrm{L}^{-1}\left(28-168 \mu \mathrm{g} \cdot \mathrm{L}^{-1}\right)$ of entry and $57 \mu \mathrm{g} \cdot \mathrm{L}^{-1}(16-$ $\left.196 \mu \mathrm{g} \cdot \mathrm{L}^{-1}\right)$ after $1 \mathrm{yr}$.

Table 3. - Individual data on upper arm skin thickness and PINP values in suction blister fluid before IC treatment (0 months) and after 3 months, 6 months and 1-2 yrs

\begin{tabular}{|c|c|c|c|c|c|c|c|c|c|}
\hline \multirow{2}{*}{$\begin{array}{l}\text { Subject } \\
\text { No. }\end{array}$} & \multirow{2}{*}{$\begin{array}{l}\text { Age } \\
\text { yrs }\end{array}$} & \multicolumn{4}{|c|}{ Skin thickness $\mathrm{mm}$} & \multicolumn{3}{|c|}{ PINP in SBF $\mu g \cdot L^{-1}$} & \multirow{2}{*}{$\begin{array}{c}\text { Dose of IC } \\
\mu \mathrm{g}\end{array}$} \\
\hline & & 0 months & 3 months & 6 months & $1-2 \mathrm{yrs}$ & 0 months & 3 months & 6 months & \\
\hline \multicolumn{10}{|l|}{ Males } \\
\hline 1 & 16 & 1.46 & 1.41 & ND & ND & 500 & 628 & ND & 800 BUD \\
\hline 2 & 17 & 1.95 & 1.89 & 1.85 & 1.96 & 1018 & 328 & 308 & 800 BUD \\
\hline 3 & 18 & 1.39 & 1.40 & 1.28 & 1.54 & 4580 & 1158 & 200 & 800 BUD \\
\hline 4 & 23 & 1.83 & 1.82 & 1.69 & 2.08 & 358 & 380 & 110 & 800 BUD \\
\hline 5 & 24 & 1.83 & 1.59 & 1.58 & ND & 793 & 110 & 168 & 800 BUD \\
\hline 6 & 27 & 1.80 & 1.78 & 1.76 & $1.55^{*}$ & 695 & 338 & 440 & 800 BUD \\
\hline 7 & 27 & 1.36 & 1.35 & 1.34 & 1.37 & 615 & 125 & 188 & 800 BUD \\
\hline 8 & 33 & 1.87 & 1.90 & 1.86 & 1.85 & 1138 & 90 & 125 & 800 BUD \\
\hline 9 & 36 & 1.81 & 1.78 & 1.49 & ND & 2360 & 593 & 1045 & 1000 BUD \\
\hline 10 & 37 & 1.47 & 1.47 & 1.59 & ND & 333 & 40 & 295 & 800 BUD \\
\hline 11 & 40 & 1.71 & 1.71 & 1.67 & $1.65^{*}$ & 1208 & 143 & 795 & 1600 BUD \\
\hline 12 & 42 & 1.37 & 1.43 & 1.41 & 1.36 & 733 & 255 & 165 & 800 BUD \\
\hline 13 & 42 & 1.71 & 1.59 & 1.50 & 1.62 & 803 & 135 & 288 & 800 BUD \\
\hline 14 & 48 & 1.93 & 1.95 & 1.73 & 1.74 & 1120 & 108 & 78 & 800 BUD \\
\hline 15 & 50 & 1.56 & 1.69 & 1.61 & 1.60 & 870 & 455 & 270 & 800 BUD \\
\hline 16 & 50 & 1.76 & 1.74 & 1.70 & $1.65 *$ & 425 & 75 & 28 & $800 \mathrm{BDP}$ \\
\hline 17 & 50 & 1.90 & 1.91 & 1.87 & ND & 70 & 500 & 315 & 800 BUD \\
\hline 18 & 64 & 1.62 & 1.70 & ND & ND & 340 & 83 & ND & 800 BUD \\
\hline 19 & 68 & 1.73 & 1.37 & 1.37 & ND & 498 & 133 & 155 & 800 BUD \\
\hline Median & 37 & 1.73 & 1.70 & 1.67 & 1.64 & 733 & 143 & 200 & \\
\hline \multicolumn{10}{|l|}{ Females } \\
\hline 1 & 17 & 1.22 & 1.20 & 1.14 & 1.32 & 205 & 178 & 63 & 800 BUD \\
\hline 2 & 32 & 1.41 & 1.25 & 1.38 & 1.16 & 230 & 108 & 40 & 800 BUD \\
\hline 3 & 35 & 1.49 & 1.53 & 1.46 & 1.51 & 323 & 138 & 70 & 800 BUD \\
\hline 4 & 37 & 1.23 & 1.24 & 1.26 & 1.06 & 113 & 78 & 183 & 800 BUD \\
\hline 5 & 41 & 1.57 & 1.36 & 1.46 & 1.49 & 273 & 95 & 110 & $1000 \mathrm{BDP}$ \\
\hline 6 & 44 & 1.47 & 1.34 & 1.34 & $1.24 *$ & 325 & 118 & 80 & 800 BUD \\
\hline 7 & 49 & 1.41 & 1.37 & 1.41 & $1.16^{*}$ & 265 & 55 & ND & 800 BUD \\
\hline 8 & 66 & 1.14 & 1.15 & 1.11 & $1.02 *$ & 208 & 110 & 55 & $1000 \mathrm{BDP}$ \\
\hline Median & 39 & 1.41 & 1.30 & 1.36 & 1.20 & 237 & 109 & 95 & \\
\hline
\end{tabular}

*: The patient received one or more courses of oral corticosteroids after a 6 month follow-up. ND: not determined. BUD: budesonide; BDP: beclomethasone dipropionate; IC: inhaled corticosteroid; SBF: suction blister fluid; PINP: aminoterminal propeptide of type I procollagen. 


\section{Collagen synthesis and degradation markers in serum}

PINP had decreased by $13 \%$ (95\% CI 1-23\%, $\mathrm{p}=0.033)$ and ICTP by $12 \%(95 \%$ CI $0-23 \%, \mathrm{p}=0.057)$ at 3 months. PINP showed an average decrease of $13 \%$ (95\% CI ranged from a $29 \%$ decrease to a $6 \%$ increase) at 6 months, but this was not statistically significant $(p=0.16)$. ICTP behaved similarly. The mean decrease of ICTP was $12 \%$ (95\% CI ranged from a $26 \%$ decrease to a $4 \%$ increase) at 6 months, and this decrease was also not statistically significant ( $\mathrm{p}=0.13$ ) (fig. 1 ).

The PICP concentration in serum was unchanged during the 6 months (data not shown).

PIIINP had decreased by $22 \%(95 \%$ CI $11-32 \%, \mathrm{p}=$ $0.001)$ at 3 months and by $20 \%(95 \% \mathrm{CI} 4-34 \%, \mathrm{p}=0.023)$ at 6 months, and no further decrease was hence observed.
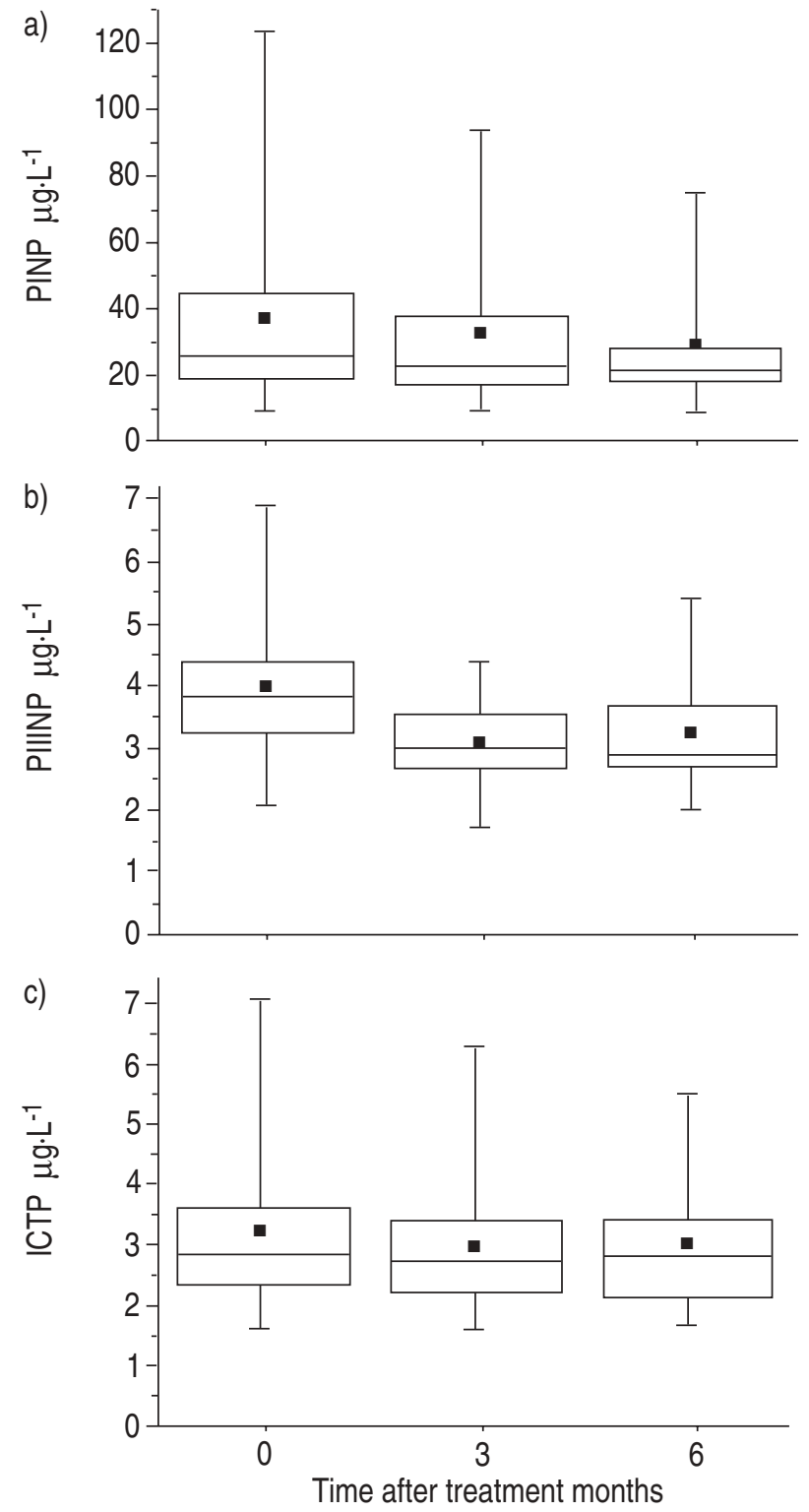

Fig. 1. - Concentrations of a) PINP; b) PIIINP; and c) ICTP in serum samples. A slight decrease of PINP and ICTP was observed at 3 months, but at 6 months the downward tendency was not so obvious. PIIINP was decreased at both 3 and 6 months. : mean. Horizontal lines represent the median, boxes represent the interquartile range and whiskers represent the range. PINP, PIIINP: aminoterminal propeptides of type I and III procollagens, respectively; ICTP: cross-linked carboxyterminal telopeptide of type I collagen.
The procollagen propeptides, PINP, PIIINP, PICP and ICTP, were analysed in serum of female controls. During the 6 month follow-up, the level of the propeptides was unchanged. The median (range) level of PINP in serum was $44.4 \mu \mathrm{g} \cdot \mathrm{L}^{-1}\left(18-101 \mu \mathrm{g} \cdot \mathrm{L}^{-1}\right)$ at entry and $46.7 \mu \mathrm{g} \cdot \mathrm{L}^{-1}$ (18.5-103.9 $\left.\mu \mathrm{g} \cdot \mathrm{L}^{-1}\right)$ after 6 months. The median (range) level of PIIINP was $3.5 \mu \mathrm{g} \cdot \mathrm{L}^{-1}\left(2.2-11.5 \mu \mathrm{g} \cdot \mathrm{L}^{-1}\right)$ at entry and $3.5 \mu \mathrm{g} \cdot \mathrm{L}^{-1}\left(3-11.6 \mu \mathrm{g} \cdot \mathrm{L}^{-1}\right)$ after 6 months. The median (range) level of PICP was $107.7 \mu \mathrm{g} \cdot \mathrm{L}^{-1}(74.5-191.8$ $\left.\mu \mathrm{g} \cdot \mathrm{L}^{-1}\right)$ at entry and $114.1 \mu \mathrm{g} \cdot \mathrm{L}^{-1}\left(63.4-198.5 \mu \mathrm{g} \cdot \mathrm{L}^{-1}\right)$ after 6 months. The median (range) level of ICTP was $2.7 \mu \mathrm{g} \cdot \mathrm{L}^{-1}$ $\left(1.7-5.4 \mu \mathrm{g} \cdot \mathrm{L}^{-1}\right)$ at entry and $2.9 \mu \mathrm{g} \cdot \mathrm{L}^{-1}\left(1.9-4.7 \mu \mathrm{g} \cdot \mathrm{L}^{-1}\right)$ after 6 months. There are no data of PINP, PIIINP and ICTP of male controls.

\section{Discussion}

We demonstrated here that the moderate IC doses generally used for the treatment of asthma are able to decrease skin and bone collagen synthesis. As the measurement of skin thickness and the total amount of skin collagen shows, use of ICs alone does not lead to marked thinning of the skin.

The study was planned to take place in a real clinical situation, and the patients were recruited into the study on the basis of the diagnosis of asthma and the initiation of treatment with ICs. In Finland, all adult asthma patients are treated with continuous ICs as a first-line treatment (except the mildest forms of asthma), and thus no untreated asthma control group was available.

We have previously measured the collagen propeptide levels reflecting collagen synthesis in vivo in patients aged 20-80 yrs with the method used in the present study, and the levels were of the same order as the pretreatment values of the present subjects [15]. In contrast, the levels noted during the IC treatment were clearly lower than the corresponding control levels recorded with the same method earlier [15]. In addition, we have previously shown that short-term treatment of young asthmatics with nedocromil does not decrease the levels of skin collagen synthesis [2]. Furthermore, we followed up the collagen synthesis of 14 healthy female volunteers for 6 months and eight male volunteers for 12 months and saw no decreases in propeptide levels. We are hence confident that collagen synthesis is decreased by ICs.

Female skin thickness seems to be smaller than male skin thickness. In the present study, the upper arm skin was about $20 \%$ thinner in females than in males, a finding in agreement with the previous studies [16]. There are data to suggest that females are more likely to develop skin bruising [17]. This finding was partially supported by our study, especially the results obtained after 1-2 yrs of treatment with IC plus oral corticosteroid, since the skin thickness of the upper arm had decreased from a median of 1.41 to $1.20 \mathrm{~mm}$. The corresponding decrease in males was from 1.73 to $1.67 \mathrm{~mm}$.

Since older patients seem to get skin bruising more often than younger patients [17], it would be interesting to compare young and old asthma patients separately. Unfortunately, most of the present asthma patients studied were middle-aged, and the data are hence not sufficient for statistical analysis. 
CAPEWEl et al. [3] demonstrated that high-dose use of ICs for $4 \mathrm{yrs}$ is associated with purpura and dermal thinning. However, a confounding influence may have been caused by previous courses of oral prednisolone. No such changes were seen in the patients who had received low-dose ICs [3]. Thus, if the IC treatment is combined with prednisolone perorally, the increased dose probably leads to skin thinning, as it did in these patients. However, the limited number of patients did not permit us to draw firm conclusions.

In previous studies, PICP has been used as a marker of bone collagen synthesis [7] and ICTP as a marker of bone degradation [12]. The previous results on the effect of ICs on bone collagen metabolism have been quite contradictory. It has been postulated that ICs may have a short-term effect on bone metabolism, but the changes are balanced in long-term use $[18,19]$. Bone resorption has been studied as well, and the results are contradictory. However, osteocalcin has been used in several studies, but this parameter does not accurately reflect bone formation [19]. In this study, we used serum procollagen propeptides as a marker of de novo synthesis of collagen, and found that they were decreased in patients on IC therapy. The degradation marker of type I collagen (ICTP) in serum decreased almost to the same extent as the synthesis marker PINP in serum, suggesting that the degradation of bone collagen may also be decreased by ICs in vivo.

Unfortunately, we are not able to measure type I collagen degradation in SBF, but the unchanged skin thickness confirms that the decrease of synthesis does not lead to a thinning of the skin after the use of moderate doses of ICs. This is possibly due to the fact that the degradation and turnover of collagen slow down, so that the net amount of collagen remains relatively constant. This assumption is supported by numerous cell culture studies, which have shown that corticosteroids decrease the activities of matrix metalloproteinases, i.e. enzymes that degrade collagens [20]. This could also explain partially why even a marked decrease in the de novo synthesis of collagen in soft tissues, including skin, did not result in a significant change in the amount of collagen.

The aim of this study was to evaluate the effects of ICs alone on skin and bone. Skin thickness was therefore measured after 1-2 yrs in all of the present patients who consented and their treatments were carefully analysed. Six patients had used oral corticosteroids after the initial 6 month study period. We found skin thickness to be decreased in the asthmatics who had received both ICs and oral corticosteroids on a periodical basis. There thus seems to be a patient group receiving both oral corticosteroids and ICs at enhanced risk for skin atrophy. This finding is in accordance with the study of CAPEWELL et al. [3].

In conclusion, patients receiving only moderate doses of inhaled corticosteroids need not generally be monitored for skin atrophy. However, possible skin atrophy should be monitored in certain high-risk individuals, i.e. patients receiving inhaled plus oral corticosteroids periodically, and at certain body sites such as arms.

Acknowledgement: The authors wish to acknowledge the expert technical assistance of K. Apajalahti.

\section{References}

1. Stead RT, Cooke NJ. Adverse effects of inhaled corticosteroids. Br Med J 1989; 298: 403-404.

2. Autio P, Karjalainen J, Risteli L, Risteli J, Kiistala U,
Oikarinen A. Effects of an inhaled steroid (budesonide) on skin collagen synthesis of asthma patients in vivo. Am J Respir Crit Care Med 1996; 153: 1172-1175.

3. Capewell S, Reynolds S, Shuttleworth D, Edwards C, Finlay AY. Purpura and dermal thinning associated with high-dose inhaled corticosteroids. Br Med J 1990; 300: 1548-1551.

4. Epstein EH, Munderloh NH. Isolation and characterization of $\mathrm{CNBr}$ peptides of human $[\alpha 1(\mathrm{III})]_{3}$ collagen and tissue distribution of $\left[\alpha 1(\mathrm{I})_{2}\right] \alpha_{2}$ and $[\alpha(\mathrm{III})]_{3}$ collagens. $J$ Biol Chem 1975; 250: 9304-9312.

5. Prockop DJ, Kivirikko KI, Tuderman L, Guzman NA. The biosynthesis of collagen and its disorders. $N$ Engl $J$ Med 1979; 301: 13-23, 77-85.

6. Melkko J, Kauppila S, Niemi S, et al. Immunoassay for the intact aminoterminal propeptide of human type I procollagen (PINP). Clin Chem 1996; 42: 947-954.

7. Melkko J, Niemi S, Risteli J, Risteli L. Radioimmunoassay of the carboxyterminal propeptide of human type I procollagen. Clin Chem 1990; 36: 1328-1332.

8. Risteli J, Niemi S, Trivedi P, Mäentausta P, Mowat AP, Risteli L. Rapid equilibrium radioimmunoassay for the amino-terminal propeptide of human type III procollagen. Clin Chem 1988; 34: 715-718.

9. Oikarinen A, Autio P, Kiistala U, Risteli L, Risteli J. A new method to measure type I and III collagen synthesis in human skin in vivo: demonstration of decreased collagen synthesis after topical glucocorticoid treatment. $J$ Invest Dermatol 1992; 98: 220-225.

10. Autio P, Oikarinen A, Melkko J, Risteli J, Risteli L. Systemic glucocorticoid treatment decreases the synthesis of type I and III collagens in human skin in vivo, whereas isotretinoin has little effect. Br J Dermatol 1994; 131: 660-663.

11. American Thoracic Society. Standards for the diagnosis and care of patients with chronic obstructive pulmonary disease (COPD) and asthma. Am Rev Respir Dis 1987; 136: 224-244.

12. Kiistala U. Suction blister device for separation of viable epidermis from dermis. J Invest Dermatol 1968; 50: 129-137.

13. Risteli J, Elomaa I, Niemi S, Novamo A, Risteli L. Radioimmunoassay for the pyridolone cross-linked carboxyterminal telopeptide of type I collagen: a new serum marker of bone collagen degradation. Clin Chem 1993; 39: 635-640.

14. Kivirikko KI, Laitinen O, Prockop DJ. Modification of a specific assay for hydroxyproline in urine. Anal Biochem 1962; 19: 249-255.

15. Autio P, Risteli J, Haukipuro K, Risteli L, Oikarinen A. Collagen synthesis in human skin in vivo: modulation by aging, ultraviolet B irradiation and localization. Photodermatol Photoimmunol Photomed 1994; 11: 1-5.

16. Tan CY, Statman B, Marks R, Payne PA. Skin thickness measurement by pulse ultrasound: its reproducibility, validation and variability. Br J Dermatol 1982; 106: 657-667.

17. Roy A, Leblanc C, Paquette L, et al. Skin bruising in asthmatic subjects treated with high doses of inhaled steroids: frequency and association with adrenal function. Eur Respir J 1996; 9: 226-231.

18. Sorva R, Turpeinen M, Juntunen-Backman K, Karonen S-L, Sorva A. Effects of inhaled budesonide on serum markers of bone metabolism in children with asthma. $J$ All Clin Immunol 1992; 90: 808-815.

19. Kerstjens HAM, Postma DS, van Doormaal JJ, et al. Effects of short term and long term treatment with inhaled corticosteroids on bone metabolism in patients with airways obstruction. Thorax 1994; 49: 652-656.

20. Oikarinen AI, Uitto J, Oikarinen J. Glucocorticoid action on connective tissue: from molecular mechanisms to clinical practice. Med Biol 1986; 64: 221-230. 